\title{
Adaptação transcultural da Dementia Management Strategies Scale ao português brasileiro
}

\section{Transcultural adaptation of the Dementia Management Strategies Scale to Brazilian Portuguese}

\author{
Marília da Nova CRUZ \\ Amer Cavalheiro HAMDAN' \\ Rochele Paz FONSECA ${ }^{2}$
}

\begin{abstract}
Resumo
O presente estudo tem por objetivo apresentar a adaptação transcultural da Dementia Management Strategies Scale ao português brasileiro. Essa escala foi desenvolvida para verificar a frequência do uso de três tipos de estratégias de gerenciamento da demência, utilizadas por familiares cuidadores de idosos: crítica, gerenciamento ativo e encorajamento. Participaram do processo de adaptação 3 tradutores, 100 juízes não especialistas, 5 juízes especialistas e 15 cuidadores. Os procedimentos utilizados foram duas traduções independentes, elaboração de uma versão síntese das duas traduções, avaliação do instrumento pelos juízes e, por fim, a condução de estudo piloto com 15 cuidadores de idosos com demência do tipo Alzheimer. Após as adaptações realizadas em todas as etapas do processo de adaptação transcultural, constatou-se equivalência satisfatória entre as versões.
\end{abstract}

Unitermos: Cuidadores. Demência. Testes psicológicos.

\begin{abstract}
The aim of the present study is to present the transcultural adaptation of the Dementia Management Strategies Scale to the Brazilian Portuguese language. This scale was developed to verify the frequency of use of three types of management strategies in dementia, by family caregivers of elderly with this disorder. It is divided into three factors: critical, active management and encouragement. There were 3 translators, 100 non-specialist judges, 5 expert judges and 15 caregivers who participated in the pilot study. The procedures conducted were: two independent translations, elaboration of a synthetic version, evaluation of the instrument by 100 non-specialist judges and by 5 specialist judges and, finally, a pilot study conducted with 15 caregivers of elderly patients with Alzheimer's Disease. After the adaptations were made in all stages of the process of the transcultural adaptation, the equivalence between the two versions was found to be satisfactory.
\end{abstract}

Uniterms: Caregivers. Dementia. Psychological tests.

$\boldsymbol{\nabla \nabla \nabla \nabla}$

1 Psicóloga. Paraná, PR, Brasil.

2 Universidade Federal do Paraná, Programa de Pós-Graduação em Psicologia, Departamento de Psicologia. Praça Santos Andrade, 50, $2^{\circ}$ andar, Sala 217, 80060-246, Curitiba, PR, Brasil. Correspondência para/Correspondence to: A.C. HAMDAN. E-mail: <achamdan@ufpr.br>.

3 Pontifícia Universidade Católica do Rio Grande do Sul, Faculdade de Psicologia, Programa de Pós-Graduação em Psicologia. Porto Alegre, RS, Brasil.

Artigo elaborado a partir da dissertação de M.N. CRUZ, intitulada "Adaptação e validação da escala de estratégias de gerenciamento da demência". Universidade Federal do Paraná, 2011. 
Com o aumento da população idosa, ocorre um aumento das doenças crônicas, como as demências. Estima-se que a prevalência geral de demência em pessoas com idade igual ou superior a 65 anos na América Latina é de 7,1\% (Nitrini et al., 2009).

A maior parte dos cuidados prestados aos portadores da doença de Alzheimer é realizada por familiares próximos (Haley, 1997). A sobrecarga dos cuidadores familiares de pacientes com demência vem sendo frequentemente investigada na literatura, e diversas teorias são apresentadas para descrevê-la e explicá-la. As variáveis do paciente, do cuidador e do contexto do cuidado são frequentemente associadas à sobrecarga dos cuidadores. Mohamed, Rosenbeck, Lyketsos e Schneider (2010) apontam as variáveis do paciente como fatores preditores de sobrecarga e depressão dos cuidadores, especialmente a relação entre a presença de distúrbios de comportamento, a menor capacidade funcional do paciente e as duas variáveis em questão. Mougias, Politis, Lyketsos e Mavreas (2010) constataram correlação positiva entre o maior comprometimento cognitivo do paciente e maior dependência funcional e a sobrecarga do cuidador. Cuidadores mais satisfeitos com a relação pré-mórbida entre ele e o paciente com demência apresentam menor sobrecarga (Steadman, Tremont \& Davis, 2007), assim como cuidadores do sexo masculino (Takano \& Arai, 2005).

Os fatores moderadores da sobrecarga do cuidador também vêm sendo investigados nos estudos. $O$ coping e o suporte social são considerados os principais moderadores de stress do cuidador.

Coping, segundo a conceituação original de Folkman e Lazarus (1980), éum processo que se dá entre o indivíduo e o ambiente, o qual tem como função a administração da situação estressora, exigindo esforço do indivíduo para administrar as demandas externas ou internas que surgem de sua interação com o ambiente. Nesse sentido, o coping pode ser compreendido como mediador entre um estressor e o resultado desse estressor.

Segundo a teoria de Folkman e Lazarus (1980), as estratégias de coping podem ser classificadas em focadas na emoção e focadas no problema. As estratégias focadas na emoção referem-se aos esforços para regular o estado emocional do indivíduo, dirigindo-se ao nível somático da tensão emocional. Já as estratégias foca- das no problema constituem esforços para atuar na situação que originou o stress, de modo a tentar modificá-la. Segundo Lazarus e Folkman (1984), qualquer tentativa de administrar o estressor pode ser considerada uma estratégia de coping.

Diversos estudos sobre cuidadores utilizam o modelo de teoria de coping proposto por Lazarus e Folkman (1984). Nesses estudos, alguns apontam que a utilização de estratégias de coping focadas no problema estão associadas a menor sobrecarga do cuidador (Di Mattei et al., 2008; Papastavrou, Kalokerinou, Papacostas, Tsangari \& Sourtzi, 2007; Yamada, Hagihara \& Nobutomo, 2008), são utilizadas com maior frequência por cuidadores do sexo masculino e estão associadas a maiores níveis de satisfação dos cuidadores (Kristensson \& Hallberg, 2007). Por outro lado, em alguns estudos, o uso de estratégias focadas na emoção está associado a maior sobrecarga do cuidador (Di Mattei et al., 2008; Papastavrou, Kalokerinou, Papacostas, Tsangari \& Sourtzi 2007), presença de sintomas depres-sivos nos cuidadores (Powers, Gallagher-Thompson \& Kraemer, 2002) e presença de mais distúrbios de comportamento no idoso com demência (Mausbach et al., 2006).

Apesar de a maioria dos estudos apontarem a estratégia focada no problema como sendo a mais favorável, alguns trabalhos encontraram o resultado oposto, como o estudo longitudinal realizado por Cooper, Katona, Orrell e Livingston (2008), no qual foi encontrada menor morbidade psicológica em cuidadores que utilizavam as estratégias focadas na emoção, e maiores níveis de morbidade naqueles que utilizavam estratégias focadas no problema. Outro estudo apontou maior sobrecarga em cuidadores que utilizavam as estratégias focadas no problema (Parks \& Pilisuk, 1991) e, em outro, não foi encontrada nenhuma relação entre sobrecarga e a utilização de estratégias focadas no problema (Borden \& Berlin, 1990).

A falta de um consenso entre os estudos pode ser atribuída à falta de especificidade contextual dos instrumentos utilizados para avaliar o coping do cuidador (Hinrichsen \& Niederehe, 1994; Williamson \& Shultz, 1993). Um estudo de revisão crítica da literatura sobre coping e cuidadores de pacientes com quadros demenciais investigou 16 estudos acerca do tema, e concluiu que somente seis deles utilizaram instrumentos específicos para cuidadores para avaliar as estratégias de 
coping em situações de demência (Kneebone \& Martin, 2003). A partir dos resultados desse estudo de revisão, os autores recomendaram a utilização de instrumentos específicos para investigações com cuidadores de pacientes com demência.

Poucos estudos utilizam instrumentos específicos que foquem em estratégias de enfrentamento no cuidado diário com o paciente em quadro de demência (Vugt et al., 2005).

No estudo realizado por Brodaty (2007), foi estabelecido um consenso sobre instrumentos utilizados em pesquisas com cuidadores. O autor afirma que a sobrecarga do cuidador é um conceito multidimensional, pois envolve aspectos físicos, psicológicos, financeiros e sociais, sendo que para cada um desses quatro aspectos há instrumentos de medida recomendados para avaliar determinados construtos, sendo alguns instrumentos desenvolvidos especificamente para cuidadores, e outros, não. O autor menciona também os fatores que podem potencializar ou diminuir a sobrecarga do cuidador, como as estratégias de coping. Para avaliar o coping do cuidador, Brodaty (2007) recomenda quatro instrumentos: (1) Health Specific Family Coping IndexforNon-Institutional Care (HSFCl), desenvolvido por Choi, Josten e Christensen (1983), para mensurar habilidade de coping na solução de problemas de saúde do paciente, no contexto físico e psicossocial; (2) Family Crisis Oriented Personal Evaluation Scale, desenvolvida por McCubbin, Larsen e Olson (1985) para avaliar estratégias de solução de problemas utilizadas pela família em situações de dificuldade; (3) Decision-Making Confidence and Skill, desenvolvida por Brennan, Ripich e Moore (1991), para avaliar habilidades na tomada de decisões; (4) Dementia Management Strategies Scale, desenvolvida por Hinrichsen e Niederehe (1994) para avaliar diferentes estratégias de gerenciamento da demência, sendo este o único construído especialmente para cuidadores.

A Dementia Management Strategies Scale foi desenvolvida por Hinrichsen e Niederehe, (1994), com o objetivo de avaliar diferentes estratégias de gerenciamento da demência. A escala é composta por 28 itens e dividida em três fatores: encorajamento, gerenciamento ativo e crítica. Os fatores referem-se a três tipos de estratégias que um cuidador de um idoso demenciado pode utilizar nos cuidados para com o paciente. A proposta original dessa escala foi desenvolvida por Scott, Wiegand e Niederehe (1984) no Texas Research Institute of Mental Sciences (TRIMS), tendo em vista a carência e a necessidade de instrumentos específicos para avaliar estratégias de enfrentamento da demência. Pesquisadores do TRIMS constataram que as escalas de coping utilizadas em pesquisas com cuidadores não possuíam especificidade contextual suficiente para mensurar padrões cognitivos e comportamentais de interação entre cuidadores e pacientes demenciados. Para tanto, os autores desenvolveram 34 itens para avaliar diferentes estratégias de gerenciamento da demência.

Os itens foram construídos com base na literatura acerca do tema, na prática clínica dos pesquisadores e, ainda, por meio de estudos piloto com familiares de idosos com quadros demenciais. Inicialmente, os itens possuíam seis domínios: Coerção; Esquiva de conflitos; Suporte emocional; Estimulação; Estruturação ambiental e Conflito. Após análises, os autores dividiram os itens em três subescalas: Estimulação/Suporte; Autoritarismo; Prevenção de Conflitos.

Posteriormente, Hinrichsen e Niederehe (1994) reanalisaram o instrumento através de uma pesquisa com uma amostra composta por 152 cuidadores informais de pacientes com demência, utilizando a análise fatorial como um dos métodos de análise de dados. A partir dos resultados dessa análise, seis itens foram excluídos, segundo os critérios estabelecidos de que aqueles com cargas fatoriais abaixo de 0,40 seriam eliminados. Três fatores emergiram a partir da análise de Hinrichsen e Niederehe (1994): Crítica (alfa de Cronbach:0,85); Encorajamento (Alfa de Cronbach:0,80); e Gerenciamento Ativo (alfa de Cronbach: 0,77). As estratégias de crítica referem-se a atitudes autoritárias do cuidador para com o idoso; as de encorajamento referem-se a esforços do cuidador para fazer com que o paciente veja o lado bom da vida; e as estratégias de gerenciamento ativo envolvem atitudes de proteção para com o paciente. Essa versão de 28 itens, proposta por Hinrichsen e Niederehe (1994) é a versão utilizada em pesquisas que procuram avaliar estratégias de gerenciamento da demência.

Embora haja instrumentos internacionais cada vez mais específicos para a avaliação das consequências biopsicossociais para cuidadores de idosos com demência, no Brasil não parece haver nenhum instrumento 
específico disponível para avaliar estratégias de enfrentamento do cuidador de paciente em quadro demencial. Tendo em vista o aumento da população idosa e, consequentemente, da prevalência de demência, há necessidade de construção e/ou adaptação transcultural de instrumentos para avaliar estratégias de enfrentamento da demência no contexto brasileiro. Esse tipo de instrumento pode contribuir para o avanço nas pesquisas da área, uma vez que possibilita estabelecer correlações entre estratégias de gerenciamento utilizadas pelos familiares de pacientes com demência e outras variáveis do cuidador, assim como variáveis do paciente e do contexto do cuidado. No contexto da prática clínica, esse tipo de instrumento pode ser de grande valia para a avaliação de estratégias de enfrentamento utilizadas com maior frequência pelos familiares de pacientes com quadros demenciais, o que permitirá, a partir desta avaliação inicial, sugerir mudanças de comportamento e programar estratégias mais eficazes, visando à melhor qualidade de vida do cuidador e do paciente com demência. Assim sendo, esta pesquisa tem por objetivo apresentar o processo de adaptação da Dementia Management Strategies Scale (Hinrichsen \& Niedehere, 1994) ao Português Brasileiro.

\section{Método}

\section{Participantes}

A amostra deste estudo foi composta por 123 participantes. Mais especificamente, fizeram parte da amostra, conforme a etapa de adaptação: 3 tradutores; 100 juízes não especialistas, selecionados por conveniência e subdivididos em seis grupos, de acordo com a faixa etária e escolaridade; 5 juízes especialistas; e 15 cuidadores frequentadores de um programa de atenção aos familiares de pacientes com doença de Alzheimer no município de Curitiba (PR) para o estudo piloto. Foram incluídos cuidadores primários e informais de idosos com diagnóstico médico de Doença de Alzheimer Provável, que concordaram em participar do estudo e assinaram o Termo de Consentimento Livre e Esclarecido (TCLE).

Foram excluídos os cuidadores de pacientes em processo de diagnóstico, de pacientes com histórico de alcoolismo ou abuso de substâncias e de pacientes com idade inferior a 60 anos.

\section{Instrumentos}

a) Questionário de informações: para avaliar dados sociodemográficos do cuidador e do paciente, características do cuidador, do paciente e do contexto do cuidado.

b) Dementia Management Strategies Scale: a escala, composta por 28 itens, propõe-se a avaliar a frequência de três tipos de estratégias de gerenciamento da demência, sendo composta por 3 fatores (3 subescalas): 1- Encorajamento, composto por oito itens (Itens: 4- 5- 7- 8- 14- 16- 19- 23). Exemplo: Item16: Eu tentei auxiliar meu parente idoso a ver o lado bom das coisas); 2- Gerenciamento ativo, composto por nove itens (Itens: 2-3-6-9-11-15-20-27-28). Exemplo: Item 3: Eu mantive um olhar atento no que meu parente idoso estava fazendo, a fim de que pudesse resolver quaisquer situações que se tornassem problemáticas); 3- Crítica, composto por onze itens (Itens: 1- 10- 12-13-17- 18- 21 22-24- 25-26). Exemplo: Item 1: Eu culpei meu parente idoso por ter criado dificuldades). A pontuação pode variar de 1 a 5 para cada item, sendo: 1 - Nunca; 2- Raramente; 3- Algumas vezes; 4- Frequentemente; 5- A maioria das vezes. Cada subescala deve ser somada separadamente.

\section{Procedimentos}

Primeiramente, este projeto foi aprovado pelo Comitê de Ética em Pesquisa do Setor de Ciências da Saúde da Universidade Federal do Paraná, CAEE nº 0042.091.085-09, em 29 de agosto de 2009, tendo todos os participantes assinado o TCLE antes de sua inclusão na amostra.

Após autorização dos autores da escala original para adaptação transcultural ao Brasil, o estudo teve início, obedecendo às etapas abaixo descritas.

Etapa 1: Tradução do instrumento. Consistiu em duas traduções do instrumento original (em inglês) para o Português Brasileiro, realizadas de forma independente.

Etapa 2: Estabelecimento de uma versão síntese do instrumento. Consistiu na elaboração de uma versão síntese das duas traduções.

Etapa 3: Análise de juízes não especialistas. A versão síntese foi aplicada em 100 juízes não espe- 
cialistas, para verificar o grau de compreensibilidade do instrumento em pessoas com diferentes idades e escolaridades, para realizar a adequação semântica do instrumento. Os juízes foram divididos em seis grupos: grupo 1, composto por 20 participantes de 18-35 anos, com 2 a 4 anos de escolaridade; grupo 2, composto por 20 participantes de 18-35 anos, com mais de 8 anos de escolaridade; grupo 3, composto por 20 participantes de 40-55 anos, com 2 a 4 anos de escolaridade; grupo 4, composto por 20 participantes de 40-55 anos, com mais de 8 anos de escolaridade; grupo 5, composto por 20 participantes de 60-75 anos, com 2 a 4 anos de escolaridade; grupo 6, composto por 20 participantes de 60-75 anos, com mais de 8 anos de escolaridade. Os locais de recrutamento dos sujeitos foram escolas de ensino supletivo, universidades e centros de convivência para idosos. Antes de responder à pesquisa, o participante foi informado sobre os objetivos e, aceitando participar do estudo, assinou um Termo de Consentimento Livre e Esclarecido. Os participantes avaliaram o grau de compreensão dos itens do instrumento assinalando a seguinte escala:

(Não é possível compreender $/ \frac{1}{1} / \frac{1}{2} \frac{1}{3} / \frac{1}{4}$ É possível compreender)

Questões que julgassem de difícil compreensão, os participantes poderiam sugerir alterações, por escrito.

Etapa 4: Análise de dados da etapa 3. Nessa etapa, foram realizadas análises estatísticas dos resultados apresentados na etapa anterior, através do teste de correlação de Pearson, do teste Kruskal-Wallis e do teste Mann-Whitney. O Kruskal-Wallis foi aplicado para verificar questões que apresentaram diferença significativa entre os grupos $(p<0,05)$. Para verificar quais grupos apresentaram a diferença estatística apontada pelo teste Kruskal-Wallis, foi aplicado o teste Mann-Whitney.

Etapa 5: Avaliação do instrumento por juízes especialistas. Após as reformulações realizadas na etapa anterior, cinco profissionais, na condição de juízes, fizeram a avaliação do instrumento quanto à pertinência e formulação dos itens, possibilitando a verificação de evidência de validade de conteúdo da escala. A amostra de juízes foi composta por um médico geriatra, uma psicóloga gerontóloga, uma psicóloga coordenadora de grupos de cuidadores de idosos com demência e por dois neuropsicólogos. Os juízes receberam o instru- mento já com as reformulações realizadas na etapa anterior, assim como informações sobre a construção da escala e sobre os objetivos e metodologia desta pesquisa. Os juízes deram seu parecer sobre a pertinência de cada item do instrumento, respondendo se concordavam ou não com o item e fizeram as observações que julgaram necessárias.

Etapa 6: Análise de dados da etapa 5. Os itens que apresentaram concordância abaixo de 80\% entre os juízes foram adaptados, seguindo sugestões dadas por eles. Para verificar a concordância interavaliadores, foi estudado o índice Kappa: valores inferiores a 0,40 indicam concordância fraca, entre 0,40 e 0,75 indicam concordância regular e acima de 0,75 indicam concordância excelente.

Etapa 7: Estudo piloto. Após as adaptações realizadas nas etapas anteriores, foi conduzido um estudo piloto com a aplicação no contexto real do instrumento como um todo em sua versão semifinal, com 15 cuidadores de idosos com demência do tipo Alzheimer. Esse estudo piloto visou à verificação da compreensão semântica da instrução inicial e dos 28 itens da Escala de Estratégias de Gerenciamento da Demência (EEGD), assim como a uma estimação do tempo de aplicação. Após o cuidador ter aceitado participar do estudo e assinado o TCLE, realizou-se a aplicação da escala e do protocolo de coleta de dados. Na aplicação da EEGD, a pesquisadora, antes de perguntar a frequência com que o cuidador utilizava a estratégia, pedia que ele desse um exemplo do possível uso de determinada estratégia, visando verificar a compreensão dos itens do instrumento.

\section{Resultados}

A partir dos resultados da análise de juízes não especialistas, foram adaptados sete itens da versão síntese da EEGD. Três itens foram adaptados por apresentarem correlação fraca $(p<0,5)$ com a pontuação total (Chan, 2003): questão $12(r=0,43)$, questão $22(r=0,28)$ e questão $24(r=0,24)$. O teste Kruskal-Wallis revelou diferença estatisticamente significativa $(p<0,05)$ de compreensão entre os diferentes grupos de juízes não especialistas em quatro itens. Mediante os resultados do teste Mann-Whitney, constataram-se diferenças significativas de compreensão $(p<0,05)$ entre Grupo 1 e Grupo 
6 nas questões 8, 17, 23 e 26; entre Grupo 3 e Grupo 6 na questão 8; entre Grupo 1 e Grupo 3 na questão 23; e entre Grupo 1 e Grupo 2 na questão 26. As questões foram adaptadas através da análise qualitativa das sugestões dos juízes não especialistas, com o auxílio, quando necessário, dos autores do instrumento original (Quadro 1).

Através dos resultados da análise de juízes especialistas, foram adaptadas as questões 10 e 14, que apresentaram concordância entre os juízes inferior a 80\%. Após a adaptação, as duas questões foram submetidas novamente à análise dos cinco juízes, que concor- daram com as alterações propostas, apresentando 100\% de concordância. O acordo interjuízes foi analisado pelo teste Kappa, obtendo o índice de 0,889 ( $p<0,001)$, indicativo de concordância excelente.

Os participantes do estudo piloto $(n=15)$ eram em sua maioria do sexo feminino $(n=11)$, casados $(n=10)$, cônjuges $(n=7)$ e filhos(as) dos idosos $(n=6)$. Os participantes cuidavam do idoso com demência, em média, há 44 meses $(\mathrm{DP}=4,6)$, sendo que a maior parte da amostra despendia mais do que 15 horas diárias nesses cuidados $(n=8)$. A partir dos resultados do estudo piloto, constatou-se que os cuidadores não demons-

Quadro 1. Adaptação dos itens no processo de adaptação transcultural da Dementia Management Strategies Scale.

\begin{tabular}{|c|c|c|c|}
\hline Item original & Tradução & Adaptação & $\begin{array}{l}\text { Etapa da qual resultou a } \\
\text { adaptação }\end{array}$ \\
\hline $\begin{array}{l}\text { 8) I encouraged my older relative } \\
\text { to keep up with friends, to visit them } \\
\text { by him/herself }\end{array}$ & $\begin{array}{l}\text { Eu encorajei meu parente idoso a } \\
\text { manter contato com os amigos e a } \\
\text { visitá-los sozinho }\end{array}$ & $\begin{array}{l}\text { Eu incentivei meu parente idoso a } \\
\text { manter contato com amigos e a } \\
\text { visitá-los sozinho }\end{array}$ & $\begin{array}{l}\text { Análise de Juízes não espe- } \\
\text { cialistas }\end{array}$ \\
\hline $\begin{array}{l}\text { 10) I asked my relative to explain } \\
\text { why he/she was doing something, } \\
\text { to draw his/her attention to his/ } \\
\text { her mistakes }\end{array}$ & $\begin{array}{l}\text { Eu pedi para meu parente idoso } \\
\text { explicar por que ele estava fazendo } \\
\text { algo, com o propósito de chamar } \\
\text { sua atenção para seus erros }\end{array}$ & $\begin{array}{l}\text { Eu pedi para meu parente idoso } \\
\text { explicar por que ele estava fazendo } \\
\text { algo, com o propósito de chamar a } \\
\text { atenção dele para seus próprios } \\
\text { erros }\end{array}$ & Análise de Juízes especialistas \\
\hline
\end{tabular}

12) I was firm with my older relative, Fui firme com meu parente idoso e Fui firme com meu parente idoso e Análise de Juízes não espeand insisted that he/she live up to insisti que ele atendesse certas insisti para que ele fizesse o que eu cialistas certain expectation / have for him/ expectativas que tenho para com espero dele her ele

14) I encouraged my relative to Eu encorajei meu parente idoso a Eu incentivei meu parente idoso a Análise de Juízesespecialistas adopt a fighting, toward his/her tomar uma atitude de luta contra lutar contra suas limitações, e fazer disability, and to do as much as suas desabilidades, e fazer o máxi- o máximo por si mesmo possible for him/herself mo por si mesmo

17) I threatened my relative with Euameacei meu parente idosocom Eu alertei meu parente idoso sobre Análise de Juízes não espedesirable consequences if he/she consequências desagradáveis se ele as possíveis consequências desa- cialistas did not cooperate não cooperasse gradáveis que ele enfrentaria caso não cooperasse

22) I left the situation for a while Eu me ausentei da situação em al- Eu me ausentei da situação em al- Análise de Juízes não espewhen relating to my older relative guns momentos quando me rela- guns momentos quando o rela- cialistas got too difficult cionar com meu parente idoso se cionamento com meu parente idotornou difícil demais so ficou muito difícil

23) I tried to reason with my older Eu tentei argumentar com meu Eutentei dialogarcommeu parente Análise de Juízes não esperelative parente idoso idoso, a fim de convencê-lo a fazer cialistas algo

24) I told my relative to stop doing Eu disse a meu parente idoso para Eu disse a meu parente idoso para Análise de Juízes não espethings that caused worry because parar de fazer coisas que causam parar de fazer coisas para mim ou cialistas of what it did to me (or to other preocupação devido ao que fazem para outros membros da família, family members) para mim (ou outro membro da fa- pois estas coisas nos causam preocumília) pação

26) I tried to communicate my older Eu tentei comunicar a meu parente Eu tentei dizer a meu parente idoso Análise de Juízes não esperelative how concerned or worried idoso o quão preocupado ou oquanto eu estava preocupado ou cialistas I was about him/her incomodado eu estava com ele incomodado com ele 
traram dificuldades na compreensão dos itens do instrumento, o que pôde ser observado através das citações de exemplos de comportamentos que representavam a estratégia de gerenciamento em questão. Por exemplo, no item 2 (Eu tentei organizar situações que pudessem estimular mentalmente ou emocionalmente meu parente idoso), alguns dos exemplos de estratégias foram: "Posso levá-lo à musicoterapia"; "Incentivo a ler jornais". O tempo estimado de aplicação do instrumento foi, em média, de 15 minutos.

\section{Discussão}

A adaptação de instrumentos elaborados em outra cultura e idioma não deve se ater à simples tradução do original, ou somente à técnica de back-translation. A tradução do teste é apenas uma das etapas do processo de adaptação, tendo em vista que a tradução literal não leva em consideração as diferenças culturais (Hambleton \& Patsula, 1998). Ainda não há consenso na literatura sobre qual a melhor maneira de se realizar uma adaptação transcultural, uma vez que pouca pesquisa vem sendo feita nesse campo para determinar o que é imprescindível nesse processo (Gillemin, Bombardier \& Beraton, 1993). Entretanto, há algum tempo pesquisadores de diversas áreas temáticas vêm sugerindo que a adequação semântica também faça parte desse processo, na medida em que seu papel é tornar o instrumento compreensível aos membros da população à qual se destina (Reichenheim \& Moraes, 2007). Nascimento e Figueiredo (2002) enfatizam também a importância de um processo de adaptação transcultural ser baseado em investigações empíricas.

Weeks, Swerissen e Belfrage (2007) destacam a importância de verificar a clareza do instrumento para que se possa realizar a adaptação dos itens mal compreendidos. Diante disso, neste estudo, além das traduções independentes para o português e síntese delas, optou-se por inserir a etapa de análise de juízes não especialistas, a qual foi extremamente importante, já que através dela foi possível mensurar o grau de compreensão dos itens por sujeitos com diferentes idades e escolaridades e fazer as adequações semânticas necessárias. Com base nos resultados dessa etapa, foram adaptadas as questões que apresentavam dificuldade de compreensão.
No processo de adaptação dos itens, foi essencial a troca de informações com os autores da Dementia Management Strategies Scale. A importância do contato com os autores do instrumento a ser adaptado foi destacada por Fonseca, Parente, Côté e Joanette (2007) no estudo de adaptação da Bateria Montreal de Avaliação da Comunicação ( Bateria MAC) ao Português Brasileiro, e muito enfatizado por Biaggio (1980). Nesse sentido, o contato com os autores da escala foi fundamental, como exemplo na adaptação do item 23 "I tried to reason with my older relative", o qual na versão síntese foi traduzido como "Eu tentei argumentar com meu parente idoso". No processo de adaptação do item, foi discutida com os autores a possibilidade da substituição da palavra argumentar por dialogar. Os autores concordaram, mas sugeriram acrescentar algo que desse o sentido de persuasão, explicando que a ideia central do item seria o cuidador persuadir o paciente a fazer alguma coisa. Dessa forma, o item foi adaptado para "Eu tentei dialogar com meu parente idoso, a fim de convencê-lo a fazer algo".

As adaptações, realizadas através dos resultados da análise de juízes não especialistas, permitem que a EEGD seja um instrumento de fácil compreensão por pessoas com diferentes idades e escolaridades.

Outro ponto a ser discutido é que, além da adaptação semântica, a análise por juízes especialistas também contribuiu para a busca de evidências de validade de conteúdo, pois a apreciação de um perito permite verificar se o teste efetivamente mensura aquilo a que se propõe (Mitrushina, Boone, Razani \& D'Elia, 2005). A tarefa dos especialistas consiste em ajuizar se os itens estão se referindo ou não ao construto em questão. Uma concordância de pelo menos $80 \%$ entre os juízes pode servir de critério de decisão sobre a pertinência do item quanto ao construto a que se refere (Pasquali, 1998). Esse parâmetro foi obtido, haja vista o índice de concordância excelente entre os cinco juízes especialistas (Kappa 0,889, $p<0,001$ ).

Em relação ao estudo piloto, embora a amostra seja relativamente reduzida, ela demonstrou ser representativa, já que suas características são semelhantes àquelas da amostra do estudo original da EEGD, no que se refere aos dados sociodemográficos, grau de parentesco com o idoso e tempo médio de cuidado. Por meio do estudo piloto, foi possível verificar que a Escala 
constituiu um instrumento de fácil compreensão para a amostra, o que pôde ser verificado pelos exemplos de estratégias citadas pelos cuidadores.

Este trabalho é relevante porque possibilitou adaptar ao contexto brasileiro uma escala específica para avaliar diferentes formas de enfrentamento que podem ser utilizadas pelos cuidadores e familiares dos idosos demenciados. A EEGD pode ser uma ferramenta útil no processo de avaliação e orientação de cuidadores de idosos demenciados, assim como pode ser um instrumento complementar nos estudos nacionais sobre o tema. Entretanto, destaca-se como limitação não ter sido utilizado o procedimento de back-translation. Como perspectivas futuras para o instrumento, recomenda-se examinar empiricamente, através de análise fatorial dos 28 itens, a ocorrência dos mesmos fatores propostos por Hinrichsen e Niedehere (1994), assim como verificar a eventual ocorrência de fatores relacionados às estratégias de coping propostas por Lazarus e Folkman (1984), sejam focadas na emoção ou no problema. Há relevância na busca de parâmetros psicométricos da EEGD, como evidências de fidedignidade, validade de construto e validade de critério deste instrumento.

\section{Referências}

Biaggio, A. M. B. (1980). Desenvolvimento da forma infantil em português do Inventário de ansiedade traço-estado de Spielberger. Arquivos Brasileiros de Psicologia, 32 (3), 106-118.

Borden, W., \& Berlin, S. (1990). Gender, coping, and psychological wellbeing in spouses of older adults with chronic dementia. American Journal of Orthopsychiatry, 60 (4), 603-610.

Brennan, P. F., Ripich, S., \& Moore, S. M. (1991). The use of home-based computers to support persons living with AIDS/ARC. Journal of Community Health Nursing, 8 (1), 3-14.

Brodaty, H. (2007). Meaning and measurement of caregiver outcomes. International Psycogeriatrics, 19 (3), 363-381.

Chan, Y. H. (2003). Bioestatistics 104: correlational analyis. Singapore Medical Journal. 44 (12), 614-619.

Choi, T., Josten, L., \& Christensen, M. L. (1983). Healthspecific family coping index for noninstitutional care. American Journal of Public Health, 73 (11), 1275-1277.

Cooper, C., Katona, C., Orrell, M., \& Livingston, G. (2008). Coping strategies, anxiety and depression in caregivers of people with Alzheimer's disease. International Journal of Geriatric Psychiatry, 23 (9), 929-936.

Di Mattei, V. E., Prunas. A., Novella, L., Marcone, A., Cappa, S. F., \& Sarno, L. (2008). The burden of distress in caregivers of elderly demented patients and it relationship with coping strategies. Neurological Science, 29 (6), 383-389.

Folkman, S., \& Lazarus, R. S. (1980). An analysis of coping in a middle-aged community sample. Journal of Health and Social Behavior, 21, 219-239.

Fonseca, R. P., Parente, M. A. M. P., Côté, H., \& Joanette, Y. (2007). Processo de adaptação da bateria Montreal de avaliação da comunicação - bateria MAC - ao português brasileiro. Psicologia: Reflexão e Crítica, 20 (2), 259-267.

Guillemin, F., Bombardier, C., \& Bearton, D. (1993). Crosscultural adaptation of health-related quality of life measures: literature review and proposed guidelines. Journal of Clinical Epidemiology, 46 (12), 1417-1432.

Haley, W. (1997). The family caregiver's role in Alzheimer's disease. Neurology, 48 (5 Suppl. 6), 25-29.

Hambleton, R. K., \& Patsula, L. (1998). Adapting tests for use in multiple languages and cultures. Social Indicators Research, 45 (19), 153-171.

Hinrichsen, G. A., \& Niederehe, G. (1994). Dementia management strategies and adjustment of family members of older patients. The Gerontologist, 34 (1), 95-102.

Kneebone, I. I., \& Martin, P. R. (2003). Coping and caregivers os people with dementia. British Journal of Health Psychological, 8 (1), 1-17.

Kristensson, E. A., \& Hallber, I. R. (2007). The association between caregiving satisfaction, difficulties and coping among older family caregivers. Journal of Clinical Nursing, 16 (5), 832- 844.

Lazarus, R.S., \& Folkman, S. (1984). Stress, appraisal and coping. New York: Springer.

Mausbach, B. T., Aschbacher, K., Patterson, T. L., Ancoli-Israel, S., von Kanel, R., Dimsdale, J. E., et al. (2006). Avoidant coping partially mediates the relationship between patient problem behaviors and depressive symptoms in spousal Alzheimer caregivers. American Journal of Geriatric Psychiatry, 29 (4), 299-306.

McCubbin, H. I., Larsen, A., \& Olson, D. H. (1985). Family crisis oriented personal evaluation scales (F-COPES). In D. H. Olson (Ed.), Family inventories: inventories used in a national survey of families across the family life cycle. St. Paul: University of Minnesota Press.

Mitrushina, M., Boone, K., Razani, J., \& D' Elia, L. (2005). Use of methodological concepts in neuropsycology practice. In M. Mitrushina, K. Boone, J. Razani \& L. D' Elia. Hamdabook of normative data for neuropsychological assessment. Oxford: Oxford University Press.

Mohamed, S., Rosenbeck, R., Lyketsos, C. G., \& Schneider, L. S. (2010). Caregiver burden in Alzheimer disease: crosssctional and longitudinal patient correlates. American Journal of Geriatric Psychiatry, 18 (10), 917-927.

Mougias, A., Politis, A., Lyketsos, C. G., \& Mavreas, V. (2010). Quality of life in dementia patients in Athens, Greece: predictive factors and role of caregiver-related factors. Intenational Psychogeriatrics, 23 (3), 1-9.

Nascimento, E., \& Figueiredo, V. L. M. (2002). WISC-III e WAIS-III: alterações nas versões originais americanas decorrentes das adaptações para uso no Brasil. Psicologia: Reflexão e Crítica, 15 (3), 606-612. 
Nitrini, R., Bottino, C. M. C., Alabala, C., Capunay, N. S. C., Ketzoian, C., Llibre Rodriguez, J. J., et al. (2009). Prevalence of dementia in Latin America: a collaborative study of population-based cohorts. International Psychogeriatric, $21,622-630$

Papastavrou, E., Kalokerinou, A., Papacostas, S. S., Tsangari, H., \& Sourtzi, P. (2007). Caring for a relative with dementia: family caregiver burden. Journal of Advanced Nursing, 58 (3), 446-457

Parks, S. H., \& Pilisuk, M. (1991). Caregiver burden: gender and the psychological costs of caregiving. American Journal of Orthopsychiatry, 61 (4), 501-509.

Pasquali, L. (1998). Princípios de elaboração de escalas psicológicas. Revista de Psiquiatria Clínica, 25 (5), 206-213.

Powers, D. V., Gallagher-Thompson, D., \& Kraemer, H. C. (2002). A longitudinal examination of coping and depression in Alzheimer's caregivers. Journals of Gerontology Series B: Psychological Sciences, 57B, 205-211.

Reichenheim, M. E., \& Moraes, C. L. (2007). Operacionalização de adaptação transcultural de instrumentos de aferição usados em epidemiologia. Revista de Saúde Pública, 41 (4), 665-73.

Scott, J., Wiegand, C., \& Niederehe, C. (1984). Measuring behavioral problems and dimensions of family caregiving in senile dementia. In $37^{\text {th }}$ Annual Scientific Meeting of The Cerontological Society of America, San Antonio, Texas.
Steadman, P. L., Tremont, G., \& Davis, J. D. (2007). Premorbid relationship satisfaction and caregiver burden in dementia caregivers. Journal of Geriatric Psychiatry and Neurology, 20 (2), 115-119.

Takano, M., \& Arai, H. (2005). Gender diffrence and caregivers' burden in eraly-onset Alzhimer's Disease. Psychogeriatrics, 5 (3), 73-77.

Vugt, M. E., Stevens, F., Aalten, P., Lousberg, R., Jaspers, N., \& Verhey, F. R. J. (2005). A prospective study of the effects of behavioral symptoms on the institutionalization of patients with dementia. International Psychogeriatrics, 17 (4), 577-589.

Weeks, A., Swerissen, H., \& Belfrage, J. (2007). Issues, challenges and solutions in translating study instruments. Evaluation Review, 31 (2), 153-165.

Williamson, G. M., \& Shultz, R. ( 1993). Coping with specific stressors in Alzheimer's disease caregiving. The Gerontologist, 33 (6), 747-755.

Yamada, M., Hagihara, A., \& Nobutomo, K. (2008). Coping strategies, care, manager support and mental health outcome among Japanese family. Helth \& Social Care in the Community, 16 (4), 400-409.

Recebido em: 23/2/2011

Versão final em: 22/3/2012

Aprovado em: 25/4/2012 
